\title{
True-Time-Delay Mechanical Phase Shifter in Gap Waveguide Technology for Slotted Waveguide Arrays in Ka-band
}

\author{
Daniel Sánchez-Escuderos, Member, IEEE, Jose I. Herranz-Herruzo, Member, IEEE, \\ Miguel Ferrando-Rocher, Member, IEEE, Alejandro Valero-Nogueira, Senior Member, IEEE,
}

\begin{abstract}
This paper proposes a novel all-metal mechanical phase shifter in gap waveguide technology. The phase shifter is aimed at providing beam-scanning capabilities to conventional slot array antennas along the elevation plane. To validate experimentally the beam-steering functionality, a $4 \times 8$ slot-array antenna has been designed and fabricated, along with the phaseshifting mechanism. The whole antenna consists of two pieces: a lower rotatable block, which changes the length of concentric Groove Gap Waveguides, and an upper fixed block, where the slot-array antenna is placed. Experimental results validate the proposed concept, having obtained steering angles of up to $25^{\circ}$, with gain levels around $20 \mathrm{dBi}$ with an antenna efficiency close to $90 \%$. A reflection coefficient below $-10 \mathrm{~dB}$ is achieved for a wide range of rotation angles from $29.5 \mathrm{GHz}$ to $30.5 \mathrm{GHz}$. The proposed phase shifter is completely scalable to any array size and its true-time-delay nature enables wide steering ranges for closely-spaced slot arrays with wideband radiation performance.
\end{abstract}

Index Terms-beam scanning, Gap-waveguide technology, phase shifter, reconfigurable antenna, satellite communications, slot array.

\section{INTRODUCTION}

B EAM-STEERING methods for high-gain antennas at millimeter-wave (mm-wave) band have been the subject of intense research over the last decade. This investigation has been largely encouraged by the deployment of new broadband mm-wave communication systems, such as $5 \mathrm{G}$ mobile networks or Ka-band satellite links, called to satisfy the everincreasing data rate usage of current users. In these systems, the severe propagation losses makes it mandatory to ensure an stable high-gain beam able to compensate for user movement. The massive adoption of these technologies, therefore, requires the development of novel cost-effective mm-wave high-gain antennas with beam scanning capabilities.

Mechanically-steered reflector antennas have been traditionally employed to establish a satellite link on mobile platforms. In addition to their efficiency, simplicity and low cost, these solutions preserve their good electrical performance for all scanning angles. Such systems, however, are often too bulky for certain applications, although the use of array antennas

This work was supported by the Spanish Ministry of Economics and competitiveness under project TEC2016-79700-C2-1-R

D. Sánchez-Escuderos, Jose I. Herranz-Herruzo, Miguel Ferrando-Rocher, and Alejandro Valero-Nogueira are with the Instituto de Telecomunicaciones y Aplicaciones Multimedia (ITEAM) of the Universitat Politècnica de València, c/ Cami de Vera s/n, 46022 Valencia, Spain (e-mail: miguel.ferrando@ua.es)

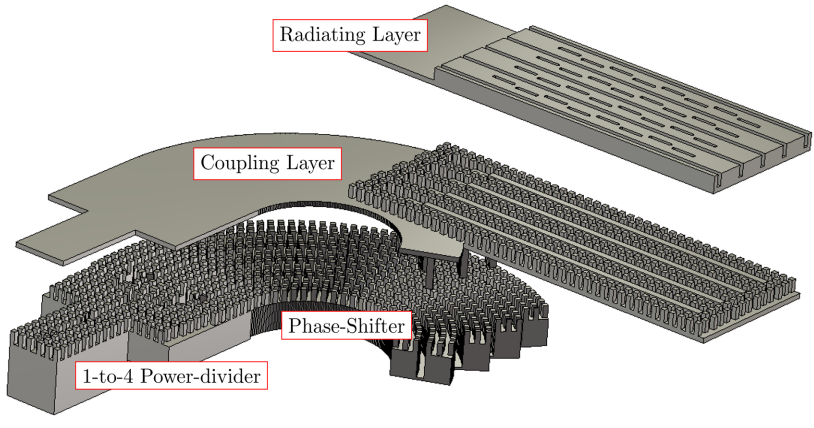

(a)

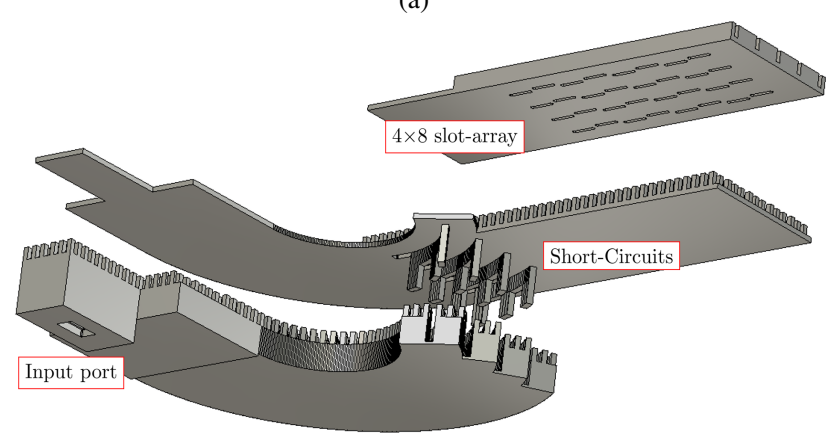

(b)

Fig. 1: Exploded view of the all-metal mechanical phase shifter in gap waveguide technology feeding a slot array antenna: (a) Top perspective view showing each of the metal pieces that make up the demonstrator, i.e., the phase-shifter, the coupling layer and the radiating layer; and (b) bottom perspective view where some important details of the layout can be observed: the input port, the phase-shifter short-circuits, and the slots.

may provide lower profiles [1]. Besides, the mechanical engines needed to steer the beam must move heavy structures without compromising their accuracy, reliability and durability.

When low profile specification is crucial, beam-steering planar arrays emerge as the most appropriate solution. In particular, active phased-arrays [2] are potentially the best suited in terms of reconfiguration versatility and speed. At the $\mathrm{mm}$-wave band, however, such fully integrated antennas suffer from high power consumption and expensive scalability. As an alternative, electronically controlled devices on printed radiators have been proposed to construct reconfigurable arrays [3].

Passive electromechanical beam scanning methods represent an appealing alternative for those applications where a very 
fast tracking is not needed. Compared to electronic steering, an electromechanical mechanism sacrifices speed and versatility in exchange for lower cost, higher efficiency and power handling. Following this general concept, several mm-wave beam-steering arrays have been recently proposed, mostly devoted to Ka-band satellite communications.

One family of solutions achieves beam scanning by the relative movement of printed phase-shifting surfaces (PSS) or lenses over a primary feed. Firstly, as with parabolic reflectors, a mere translation or rotation of the feed produces the desired beam scanning performance [4]-[7]. Note that these solutions, as well as many others mentioned later, provide one-dimensional beam scanning along elevation plane whereas full azimuth sweep would be achieved by the antenna rotation. Secondly, drawing on the so-called Risley prism concept, a two-dimensional beam scanning can be attained by rotating two PSS over a primary feed [8]. This idea was later extended [9], [10] to be able to transform the near field of a fixed-beam antenna, thus reducing the total structure height.

A second class of approaches keeps the radiating part of the antenna fixed while a small internal part is moved to steer the main beam. Such movable parts are much lighter than the entire antenna and the adhoc mechanisms needed to move them do not interfere with radiation patterns, unlike lens-based solutions mentioned above. Briefly, these approaches steer the beam by conveniently modifying the phase of the propagating mode within a waveguide, which in turn excites an array composed of slot-like radiators. The required phase control can be realized by changing either the mode propagation constant or the distance traveled by that mode.

Following the first option, several works conceived slot arrays fed by partially dielectric-filled waveguides. A movable dielectric plunger [11] or two rotating dielectric slabs [12] were proposed to modify the propagation constant of the fundamental mode within a parallel-plate waveguide (PPW) and a rectangular waveguide (RW), respectively. In this way, when a traveling-wave slot array is excited by those reconfigurable waveguides, the main beam can be steered within a certain range of elevation angles. In both works a linear array is studied, but, nevertheless, the extension to a two-dimensional version appears not to be straightforward.

Alternatively, Continuous Transverse Stub (CTS) antennas allow to modify the phase profile of the illuminating PPW mode by displacing or rotating the primary source [13][15]. In this case, the phase control relies on the different distance traveled by the electromagnetic wave before reaching the radiators. A similar strategy is followed in [16] but using circularly-arranged long slots as radiators. The angle formed by the top radiating piece and the bottom feeding piece governs the phase profile on the slots and, therefore, the main beam direction. Rotation between both pieces are enabled thanks to the contactless nature of the Gap Waveguide (GW) technology [17] in which feeding network is implemented. Such highly symmetric scheme provides a wideband and large coverage beam-steering capability, as reported in [16].

By contrast, instead of modifying the phase of a continuous wavefront, a discrete approach can be adopted by guiding the wave along independent paths of variable length. In [18], such reconfigurable paths are implemented by a number of arcshaped waveguide sections with linearly increasing radii at several layers. An intermediate rotatory piece controls the path difference between channels, thus behaving as a phase shifter. To enable a proper rotation between pieces, contactless hollow waveguides are employed at each layer including the radiating slot array. Thanks, respectively, to the all-metal waveguiding, and a true-time-delay (TTD) scheme, high efficiency and good linearity over frequency were demonstrated [18]. The proposed mechanism, however, adopts a quite intricate multilayer architecture which makes it difficult to extend the concept to an arbitrary number of array elements.

This paper proposes a novel all-metal mechanical phaseshifting mechanism capable to provide a conventional slot array with a beam-scanning performance along the elevation plane (see Fig. 1). Similarly to [18], the length of an arbitrary number of arc-shaped waveguides can be modified by the movement of a rotatory piece, leading to a TTD phase shifter. Compared to [18], this solution is far simpler since the beamsteering array only comprises two layers: the fixed upper layer corresponding to the waveguide slot array, and the rotatable lower layer containing the feeding waveguides. Besides, the proposed approach is completely general, and easily scalable so that it may be applied to any waveguide slot array of any size. This scheme could potentially be utilized in arrays with different operation modes, such as traveling-wave, leaky-wave or resonant arrays, either in linearly- or circularly-polarized versions. Beam-steering functionality can thus be added to the well-known advantages of slot arrays, i.e. high efficiency, good crosspolar discrimination, high power handling or easy synthesis of radiation patterns.

A conceptually similar phase-shifting mechanism was proposed in [19], but only very preliminary results were reported. To the authors' knowledge, the proposed structure in [19] is hardly implementable in practice, since it employs complicated waveguide bends and ideal removable shortcircuits. That contribution did not include any hints about how to realize such elements. In our proposal, GW technology [17] has been used to enable the free rotation of the bottom piece without any sort of field leakage, leaving a small gap between pieces. This technology has demonstrated to be a cost-effective and a highly efficient way to feed mm-wave slot arrays [20], [21].

In order to validate experimentally the beam-steering functionality, a $4 \times 8$ slot array has been designed and fabricated along with the phase-shifting mechanism, which was preliminary described in [22] and recently patented in [23]. A uniform resonant shunt slot array has been chosen because of its simple and well-known design rules. In particular, such array is hosted on Ridge Gap Waveguides (RGW) to facilitate the antenna assembly while keeping a reduced array spacing. Note that, at the top radiating layer, other waveguide types might have been used if necessary, since this piece remains fixed. The bottom phase-shifting layer, on the other hand, is implemented by vertical Groove Gap Waveguides (GGW)in order to reduce channel spacing and permit its free rotation.

The rest of the paper is organized as follows. Firstly, the mechanical phase shifter in GW technology is presented. A brief overview of the GW technology is given, and each component 
of the shifter is suitably described. Secondly, the slot array antenna designed for validation purposes is described. Later, both blocks are assembled to form the complete validation prototype. Measurements of this prototype are shown, and compared to simulated and theoretical results. Finally, the main conclusions of this work are highlighted.

\section{Mechanical Phase ShIFTER IN GAP WAVEGUIDE TECHNOLOGY}

The goal of the phase shifter is to distribute the input power from a single input port to $N$ output ports with a constant phase shift between consecutive ports (see Fig.1). The phase shifter can be designed to provide any amplitude distribution at the output ports to satisfy the specifications of the intended application. However, in this paper, we will focus on a uniform amplitude distribution at the output ports.

The phase shift is obtained in a true-time-delay fashion, by using transmission lines with different lengths for each output port. In order to control the phase shift, the mechanical phase shifter must be able to change the length of those lines without moving the position of the output ports.

The mechanical phase shifter proposed here is divided into two major blocks in order to obtain the above performance: an upper fixed block, eventually connected to the array antenna, and a lower rotatable block, in charge of configuring the length of the transmission lines. The latter block is rotated to modify the distance between the input port, and the end of the phaseshifting transmission lines. These lines will be later connected to the upper fixed block to attain the desired phase shift at the output ports.

To achieve free movement of the lower rotatable block with respect to the upper fixed block it requires a small gap between the pieces to be effectively produced. This gap would cause a large leakage of power if a conventional waveguide were used, especially at mm-wave frequencies. For that reason, GW technology [17] is employed to design the entire mechanical phase shifter. In particular, we use GGW waveguides at the lower block, and RGW waveguides for the upper block. Fig. 1 shows a schematic view of the complete phase shifter connected to the slot array antenna in GW technology.

In practice, the two blocks of the mechanical phase shifter are implemented by means of three components: a 1:N power divider, the phase-shifting waveguides, and the transitions between the two levels. Fig. 2 shows an upper view of the mechanical phase shifter, where each component is suitably labeled.

The 1: $N$ power divider is implemented in the lowerrotatable block in GGW technology. The goal of this compoment is to distribute the energy from a single input port to $N$ output ports (note that $N=4$ in Fig. 2). These output ports are connected to the second component, also in the lowerrotatable block, $N$ concentric GGW around a rotation axis, ended at shortcircuits. These shortcircuits are in fact attached to the upper block (as shown in Fig. 1b). This plane is the floor of the upper-fixed block. Therefore, when rotating the lower block, the electric length between the output ports of the power divider and the shortcircuits varies according to the rotation angle.

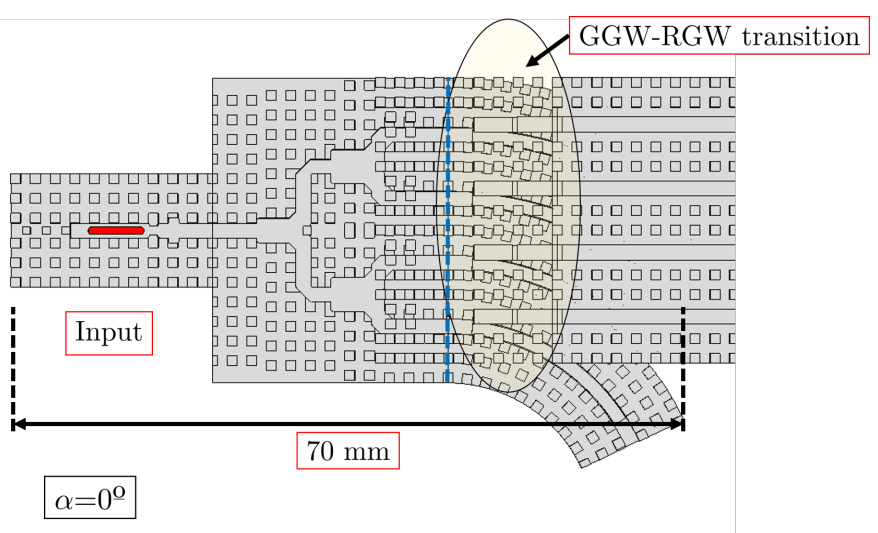

(a)

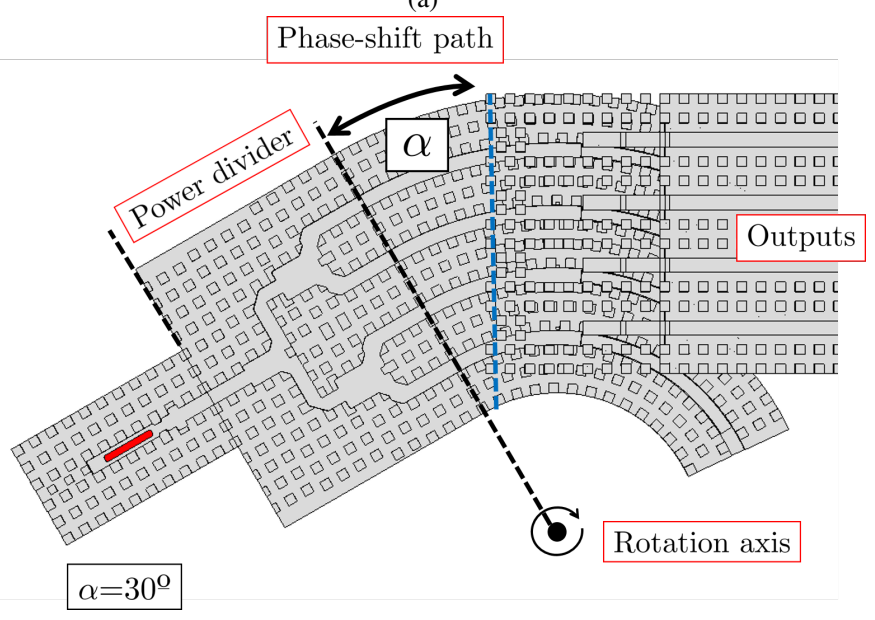

(b)

Fig. 2: Upper view of the complete phase shifter without metallic covers for a rotation angle of (a) $0^{\circ}$, i.e., all the waveguides fed with the same phase, and (b) $30^{\circ}$.
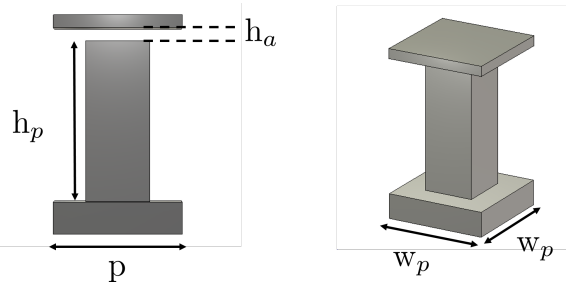

Fig. 3: Single nail of the bed of nails used for the mechanical phase shifter.

Just before the aforementioned shortcircuits, a slot is placed to connect the two blocks. This slot is part of the third component, the transition, in charge of coupling the energy from the lower GGW to the upper output RGW.

The general behavior of the mechanical phase shifter is as follows. The energy is injected at the input port, and divided into $N$ GGW with the 1:N power divider. Then, each GGW applies a different phase shift, depending on the rotation angle of the lower block. At the end of the GGW, the energy is transmitted to the upper block through a slot. These will, eventually, feed the array antenna. By suitably rotating the lower block, the phase shift at the output RGW can be dynamically modified. 


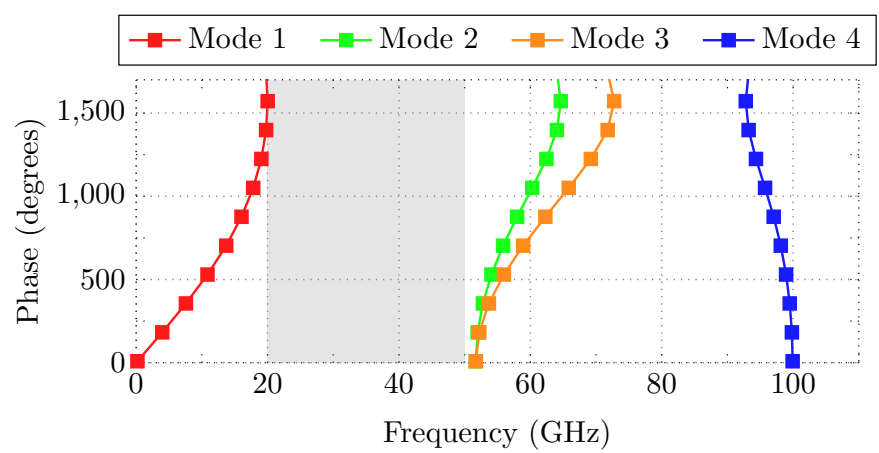

Fig. 4: Stopband of the bed of nails used in the mechanical phase shifter.

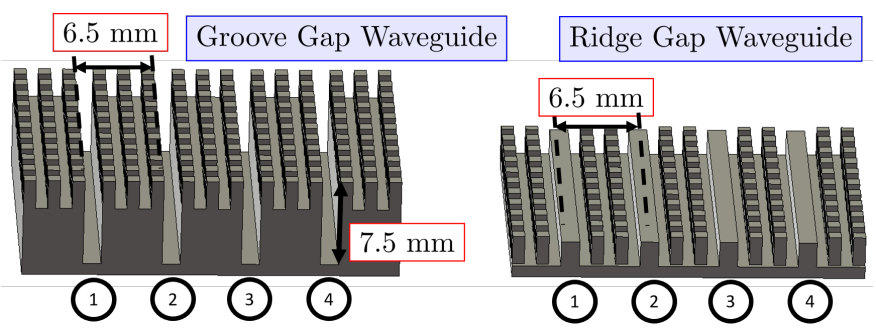

Fig. 5: 3D view of 4 parallel GGW (left) and 4 parallel RGW (right) housed in the bed of nails.

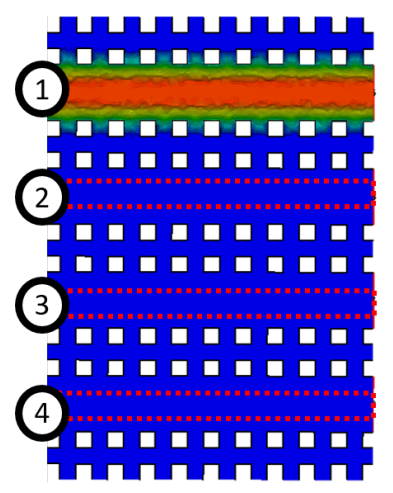

(a)

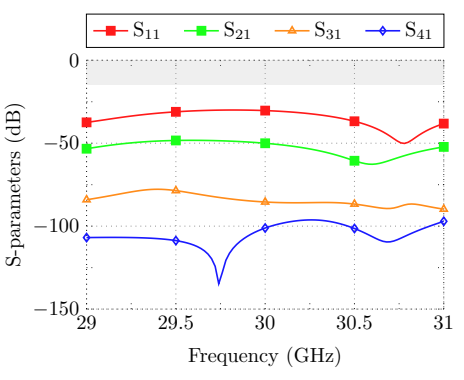

(b)
Fig. 6: (a) Representation of the electric field magnitude along the RGW and (b) simulated results of the coupling between the RGWs.

Next sections detail the main characteristics of the GW technology, explain the performance of the mechanical phase shifter, and describe each constitutive element. The operation frequency band of these elements will be $1 \mathrm{GHz}$ centered at $30 \mathrm{GHz}$, aimed to be used in Ka-band satellite communications applications.

\section{A. Gap Waveguide technology}

GW technology is commonly used to overcome the drawbacks of conventional metallic waveguides at high frequencies [24]. The main advantage of GW technology is that it does not need a perfect metallic contact between the different parts of the structure in order to avoid leakage in joints, especially critical at mm-wave frequency bands.

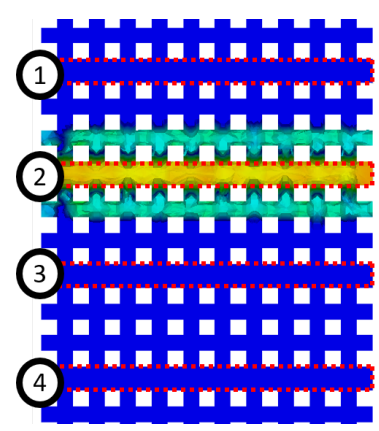

(a)

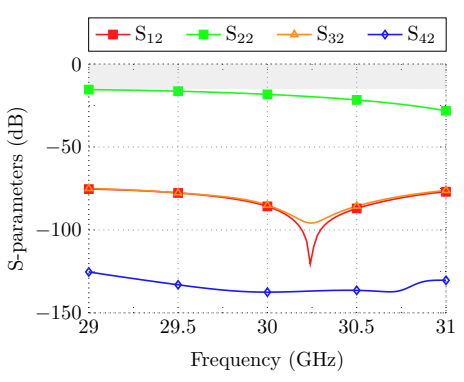

(b)
Fig. 7: (a) Representation of the electric field magnitude along the GGW and (b) simulated results of the coupling between the GGWs.

The operating principle of the GW technology consists in the creation of a stopband that can typically be one octave wide. To achieve this stopband, a bed of nails with a height of a quarter wavelength is used. Nails are commonly spaced a quarter wavelength too, though this may be a flexible criterion. Several options can be used to guide the waves in GW technology: RGWs, GGWs, inverted-microstrip gap waveguide, or microstrip-rigde gap waveguides. The operating modes propagating along these gap waveguide geometries are different. The GGW is closely equivalent to a rectangular waveguide, supporting the propagation of TE/TM modes. The fundamental mode in the RGW, the inverted-microstrip gap waveguide, and the microstrip-ridge gap waveguide is a quasiTEM mode within the stopband created by the surrounding periodic structures. Advantages and disadvantages of each version are related to the manufacturing simplicity, the compactness, and the power handling capabilities [25].

In this work, only all-metal waveguides are used (GGW and RGW). The GGW is used in the lower block, due to its suitability to easily design compact power splitters. For the upper block, RGWs are employed to enable a simple design and fabrication of closely-spaced slot arrays. The validation array will be formed by 4 parallel RGW linear slot arrays with 8 longitudinal elements each. Therefore, although the proposed concept of mechanical phase shifter can be extended to a higher number of output ports, this paper will focus on the case with 4 output RGW ports.

The separation between array rows determines the dimensions of the gap waveguides used. In this regard, a trade-off arises between the maximum scanning angle without grating lobes and the needed fabrication accuracy. Here, with the aim of validating the proposed concept, a compromise value of $0.65 \lambda_{0}$ at $30 \mathrm{GHz}$ has been chosen. This value provides a relatively wide steering range without stressing too much the fabrication process. It is envisaged to reduce this array spacing in future works. With this in mind, we need to design a bed of nails capable of providing the stopband at the operation frequency band $(29.5-30.5 \mathrm{GHz})$, and minimizing the coupling between adjacent rows.

Fig. 3 shows the geometry of one nail with its relevant dimensions. The height of the nail, $h_{p}$, is $2.5 \mathrm{~mm}$, a quarter- 




Fig. 8: Top view of the 1:4 power divider in GGW technology (metallic lid is transparent to help visualization). All the geometrical parameters of the power divider are indicated.

TABLE I: Values for all 1:4 power divider parameters.

\begin{tabular}{|c|c||c|c|}
\hline Parameter & Value $(\mathrm{mm})$ & Parameter & Value $(\mathrm{mm})$ \\
\hline \hline $\mathrm{w}_{G G W}$ & 1.5 & $\mathrm{~h}_{G G W}$ & 7.5 \\
\hline $\mathrm{w}_{b 1}$ & 2.5 & $\mathrm{l}_{b 1}$ & 3 \\
\hline $\mathrm{w}_{b 2}$ & 3.5 & $\mathrm{l}_{b 2}$ & 3.2 \\
\hline $\mathrm{w}_{c 1}$ & 7.25 & $1_{b 3}$ & 5.5 \\
\hline $\mathrm{w}_{c 2}$ & 4 & $1_{c 1}$ & 2 \\
\hline $\mathrm{w}_{p}$ & 1 & $\mathrm{~h}_{p}$ & 2.5 \\
\hline & & $\mathrm{p}$ & 2 \\
\hline
\end{tabular}

wavelength at the central frequency $\left(\lambda_{0}=10 \mathrm{~mm}\right.$ at $\left.30 \mathrm{GHz}\right)$. There is an air gap $h_{a}$ of $0.2 \mathrm{~mm}$ left between the nails and the upper lid to allow the mechanical movement between pieces. This air gap is typically chosen smaller than $0.1 \lambda_{0}$, although any air gap smaller than a quarter-wavelength would be enough to ensure the confinement of field. The width $\left(w_{p}\right)$ and the periodicity $(p)$ of nails are not as relevant as their height and the air gap, as demonstrated in [26]. Typically, $w_{p}$ is half $h_{p}$, here $1 \mathrm{~mm}$, and $p$ is twice $w_{p}$, here $2 \mathrm{~mm}$.

Fig. 4 shows the stopband obtained with the bed of nails described. We can see that the stopband ranges from 20 to $50 \mathrm{GHz}$. This bandwidth $(85.7 \%)$ is much wider than the intended operation frequency band for the mechanical phase shifter presented in this paper $(6.5 \%)$. Note also that the phase shifter reflection coefficient is lower than $-20 \mathrm{~dB}$ from $29 \mathrm{GHz}$ to $31 \mathrm{GHz}$. However, the entire system will have a narrower frequency bandwidth (3.3\%) as a result of integrating the antenna into the entire system, as will be seen below.

Once the bed of nails is chosen, we define the RGW and verify the coupling between parallel output RGW. Fig. 5 shows an overview of four parallel RGWs. The location and separation of the RGWs is constrained to the aforementioned bed of nails, and the separation between the center of ridges is $0.65 \lambda_{0}$. Note that, at least one row of nails must be used along both sides of the central ridges to produce a stopband,



(a)

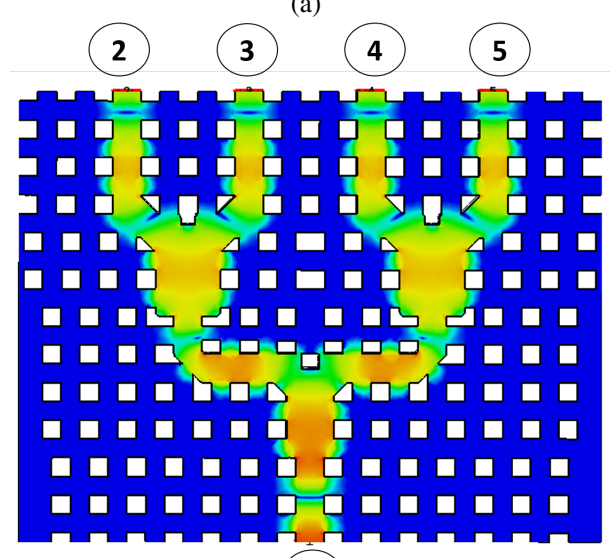

1

(b)

Fig. 9: 1:4 power divider in GGW: (a) upper view without lid, and (b) electric field magnitude at $30 \mathrm{GHz}$.

and confine the energy between the ridges and the metallic lid. It was demonstrated in [26] that one row of nails achieves approximately $20 \mathrm{~dB}$ of isolation between adjacent RGWs. This behavior is shown in Fig. 6a. We can see how an intense electric field appears in the first row of nails. Beyond the second row, the magnitude of the electric field is negligible. The isolation is greater than $60 \mathrm{~dB}$ with three rows of nails in between. However, a three-row distance would be too much for our design $\left(0.85 \lambda_{0}\right)$. A trade-off choice is to separate the parallel RGWs with two rows of nails $\left(0.65 \lambda_{0}\right)$, providing a coupling better than $50 \mathrm{~dB}$ (see Fig. 6b).

The coupling between the lower GGWs has also been studied. Fig.7a shows 4 parallel GGWs housed in the same bed of nails described above. Since the horizontally-polarized GGW is narrower than the RGW, 3 rows of nails can be used between GGWs. The isolation between adjacent GGWs is thus better than between RGWs (above $75 \mathrm{~dB}$ ), as we can see in Fig. $7 b$.

\section{B. 1:N Power divider}

The 1: $N$ power divider splits the energy into $N$ output GGW, $N$ being the number of output RGW of the mechanical phase shifter.

The power divider has a tree topology to maximize the bandwidth, and produce a uniform amplitude and phase distributions at its output ports. Figs. 8 and 9 show the power 




Fig. 10: S-parameters of the 1:4 power divider in GGW technology.

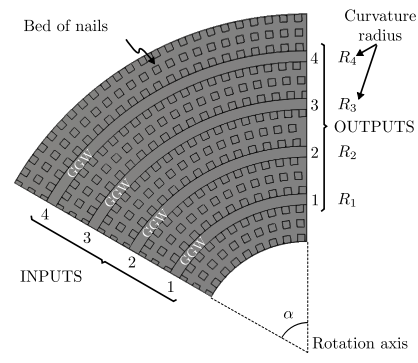

(a)

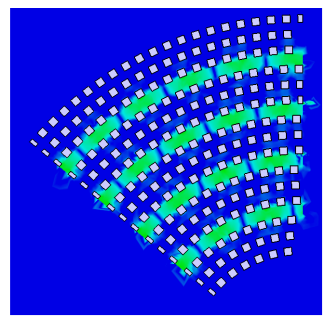

(b)
Fig. 11: Concentric phase-shifting GGWs without metallic cover: (a) upper view without lid, and (b) electric field magnitude at $30 \mathrm{GHz}$.

divider for $N=4$. GGWs are labeled as GGW in Fig. 9. As commented above, the bed of nails is intended to allow a small gap between the bed of nails of the GGW, and the metallic lid of the power divider, without leakage [27]. Note that this lid is the intermediate metallic plane that acts, in turn, as the floor of the upper-fixed block.

The optimized 1:4 power divider in GGW technology provides a good frequency response along the whole bandwidth. As we can see in Fig. 10, the $\mathrm{S}_{11}$-parameter is below $-25 \mathrm{~dB}$ along the operation frequency band, with a nearly constant insertion loss of $6 \mathrm{~dB}$. This good performance is also illustrated in the electric field shown in Fig. $9 \mathrm{~b}$.

\section{Phase-shifting waveguides}

The output ports of the $1: N$ power divider are connected to $N$ GGWs, all concentric around a rotation axis. Fig. 11a shows an upper view of $N=4$ concentric GGW without the lid. As we can see, input ports are located on a radial plane, and output ports are placed on another plane, rotated $\alpha$ with respect to the first one. Since each GGW has a different path length, the phase at the output ports will be different for each GGW. The phase shift between consecutive output ports $\left(\phi_{i, j}\right)$ depends on the rotation angle $\alpha$, and the curvature radius $R_{i}$ of each GGW as follows:

$$
\phi_{i, j}=\beta_{G G W} L_{i, j}
$$

where $\beta_{G G W}$ is the propagation constant of the GGW, and $L_{i, j}$ is length difference between GGWs:

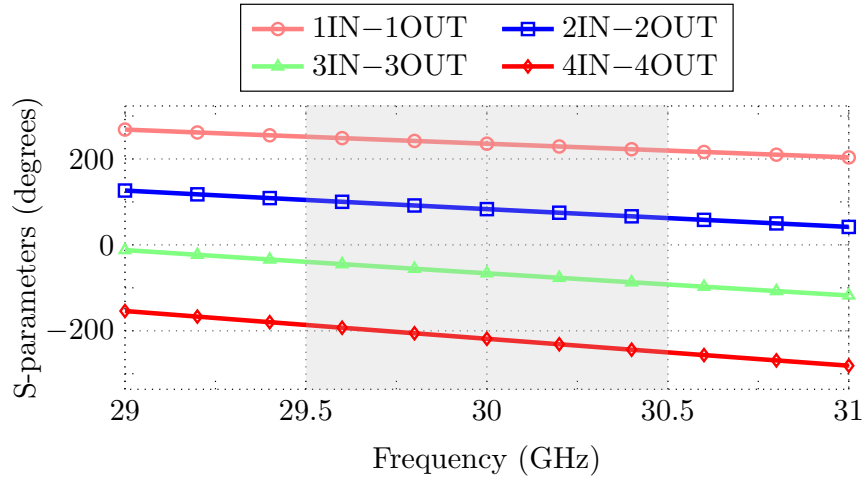

Fig. 12: Phase of the S-parameters of the concentric phaseshifting GGW for $\alpha=50^{\circ}$.

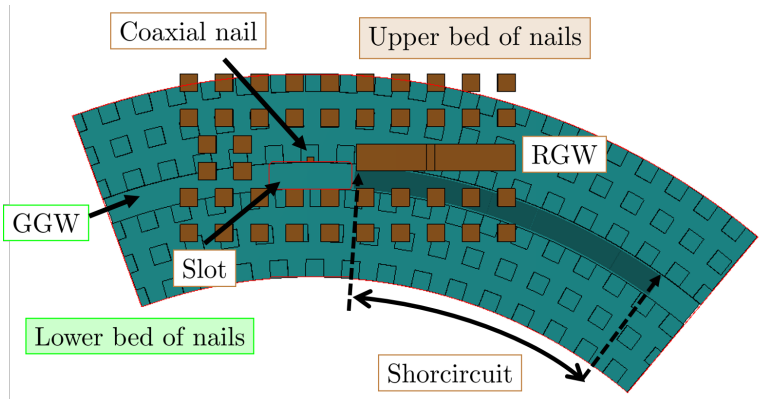

Fig. 13: Upper view of the two-level GGW-RGW transition.

$$
L_{i, j}=\left(R_{j}-R_{i}\right) \alpha
$$

Since the increment of radius is constant, the phase shift between consecutive output ports is also constant. Fig. 12 shows the phase of the S-parameters between the input and the output ports of the concentric phase-shifting GGW shown in Fig. 11a. As we can see, the phase gap between the different curves is constant. This phase shifting can also be observed in the electric field at $30 \mathrm{GHz}$ illustrated in Fig. $11 \mathrm{~b}$.

\section{GGW-RGW transition}

The previous components are assembled on the lowerrotatable block. In order to have the output ports fixed, the output RGW are placed on the upper-fixed block, as commented above. Thereby, a two-level GGW-RGW transition is necessary to transmit the energy from the output ports of the lower concentric GGW, to the upper RGW. To design this component, it must be taken into account that the intermediate fixed metallic plane is, at the same time, the lid of the GGW (separated $200 \mu \mathrm{m}$ from the GGW bed of nails) and the floor of the upper-fixed RGW, so that the lower-rotatable block does not require other lid.

The GGW-RGW transition is shown in Figs. 13-14. This transition can be split into three main parts. The first part is a concentric E-shaped metallic protrusion, shown in Fig. 13 (see black concentric box inside the GGW), and Fig. 14, acting as a shortcircuit in the GGW. E-shape protrusion acts as a filter that reflects all the power reaching the protrusion. 


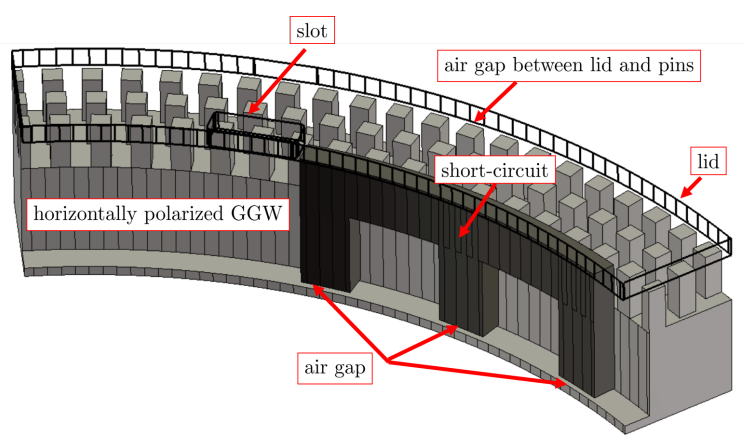

Fig. 14: 3D view of the short-circuit attached to the intermediate metallic plane between the lower and the upper blocks.

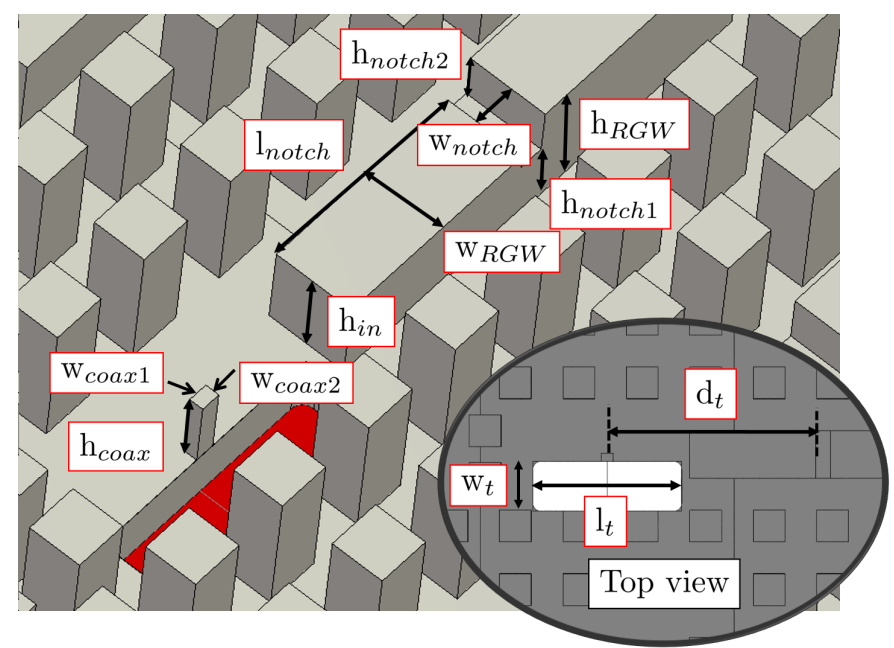

Fig. 15: 3D view of the GGW-RGW transition.

TABLE II: Geometrical parameters of the GGW-RGW transition.

\begin{tabular}{|c|c||c|c|}
\hline Parameter & Value $(\mathrm{mm})$ & Parameter & Value $(\mathrm{mm})$ \\
\hline \hline $\mathrm{w}_{\text {coax } 1}$ & 0.37 & $\mathrm{~h}_{\text {coax }}$ & 1.32 \\
\hline $\mathrm{W}_{\text {coax } 2}$ & 0.26 & $\mathrm{~h}_{\text {in }}$ & 1.6 \\
\hline $\mathrm{w}_{R G W}$ & 1.5 & $\mathrm{~h}_{R G W}$ & 2.2 \\
\hline $1_{\text {notch } 1}$ & 4 & $\mathrm{~h}_{\text {notch } 1}$ & 1.6 \\
\hline $1_{\text {notch } 2}$ & 0.46 & $\mathrm{~h}_{\text {notch }}$ & 0.9 \\
\hline $\mathrm{w}_{t}$ & 1.5 & $\mathrm{l}_{t}$ & 4.7 \\
\hline $\mathrm{d}_{t}$ & 6.5 & & \\
\hline
\end{tabular}

Physically, the protrusion is attached to the fixed upper block, and is inserted in the GGW, without touching the GGW walls. The same circular shape as the GGW enables the latter to slide when rotating the lower block, keeping the protrusion always at the same point with respect to the upper output ports, despite rotating the lower block. Thereby, this rotation varies the length of the concentric GGW, taken from the output of the power divider to the short-circuit, and, consequently, produces a variable phase shift in each GGW.

The second part of the GGW-RGW transition is a slot in the intermediate fixed metallic plane (labeled in Fig. 13). The purpose of this slot is to transmit the energy from the



Fig. 16: S-parameters of the GGW-RGW transition.

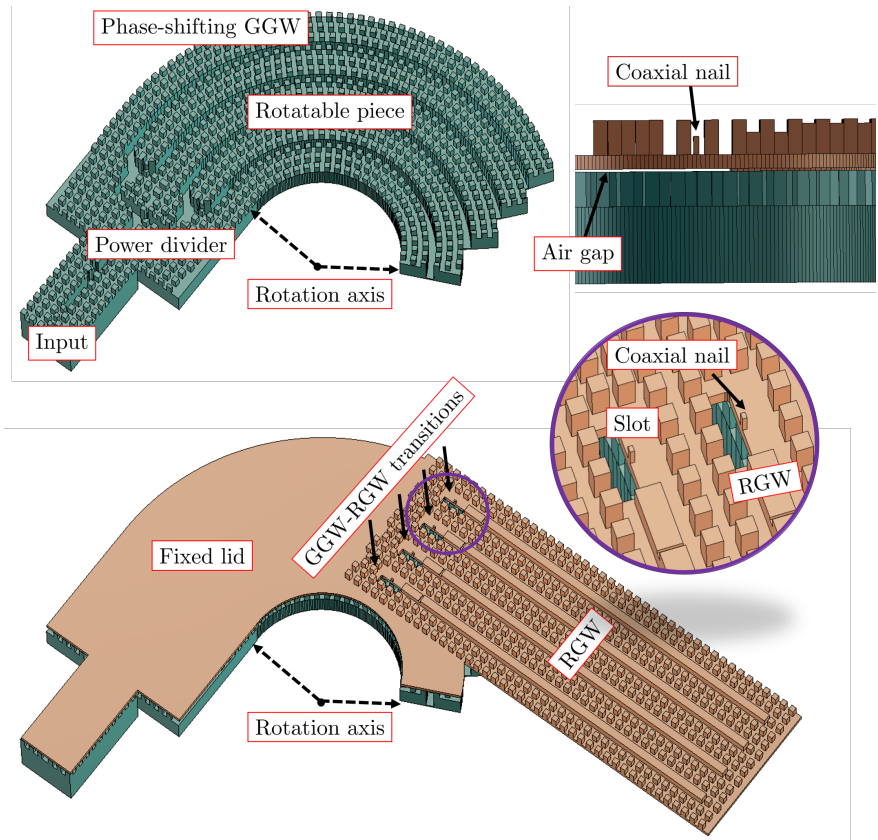

Fig. 17: 3D view of the complete phase shifter without and with the upper RGW lid.

lower-rotatable GGW, to the upper fixed RGW level. To do so, the position of the slot with respect to the shortcircuit is suitably optimized to maximize the electric field into the slot for a large bandwidth. The optimization process has been implemented by using several algorithms included in CST Microwave Studio [28], such as Trusted Region Framework and Covariance Matrix Adaptation Evolutionary Strategy.

The third part is a coaxial cavity in gap-waveguide technology on the upper face of the intermediate plane [29]. Fig. 15 shows a larger view of this cavity. This cavity is in charge of transforming an horizontally-polarized electric field in the lower GGW, into a vertically-polarized electric field (quasi-TEM mode) in the output RGW, which entails a great difficulty, especially for the considered large bandwidth. To do this transformation, the cavity has a central coaxial nail surrounded by a bed of nails, and is fed by the slot in the intermediate fixed metallic plane. This slot excites a magnetic field around the central nail in the coaxial cavity. Later, this field is coupled to the output RGW by exciting the quasi-TEM 




Fig. 18: $\mathrm{S}_{11}$-parameter of the mechanical phase shifter for several rotation angles $(\alpha)$.

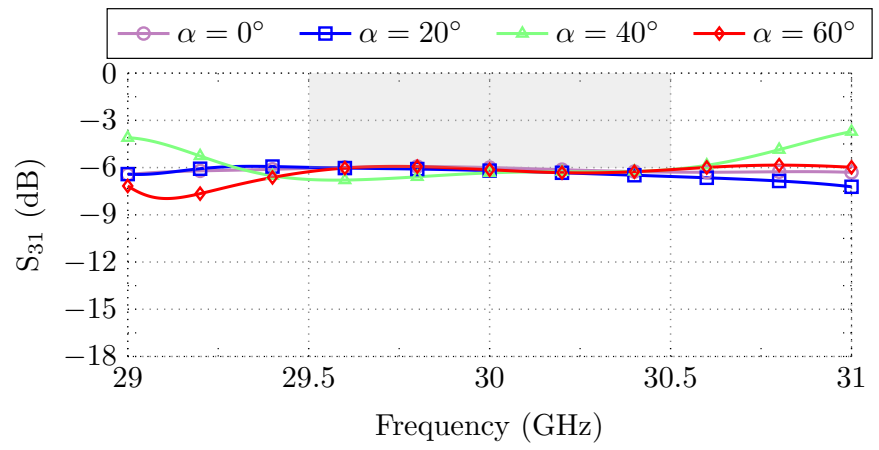

Fig. 19: $S_{31}$ parameter of the mechanical phase shifter for several rotation angles $(\alpha)$.

mode of this waveguide.

Fig. 16 shows the S-parameters of the GGW-RGW transition optimized for the mechanical phase shifter. As we can see, a return loss better than $20 \mathrm{~dB}$ is obtained for the whole operation frequency band, with a very good insertion loss between the input GGW and the output RGW.

\section{E. Complete phase shifter performance}

The three components above are assembled to form the complete mechanical phase shifter. Fig. 2 shows an upper view of the whole structure. For a better illustration, Fig. 17 shows a 3D view of the complete mechanical phase shifter. The lid of the RGW is not shown to visualize better the inner parts of the phase shifter, and the position of the different components.

Fig. 18 plots the $S_{11}$ parameter at the input port of the mechanical phase shifter for four rotation angles $\alpha$. As we can see, the return loss is better than $15 \mathrm{~dB}$ from $29.5 \mathrm{GHz}$ to $30.5 \mathrm{GHz}$ for all rotation angles, which is a good level, taking into account that output RGW will be eventually connected to an antenna. The insertion loss for all ports is around the ideal level of $6 \mathrm{~dB}$ due to the use of a 1:4 power divider, as we can see in the $S_{31}$ parameter plotted in Fig. 19 (see port labels in Fig. 8). This figure also illustrates that the insertion loss is quite flat within the whole operation frequency band.

The full performance of the mechanical phase shifter must be assessed determining the phase shift between consecutive ports, as shown in Fig. 20. As commented above, the same phase shift is ideally obtained for any pair of consecutive ports



Fig. 20: Simulated (solid lines) and expected (dashed lines) phase difference between output ports 3 and 4 for several rotation angles $\alpha$ of the mechanical phase shifter.

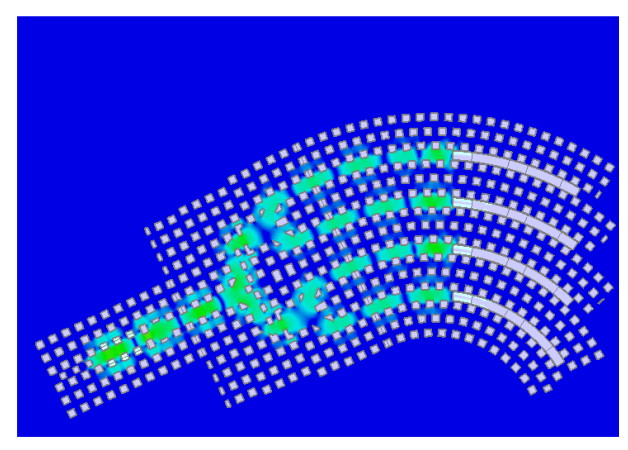

(a)

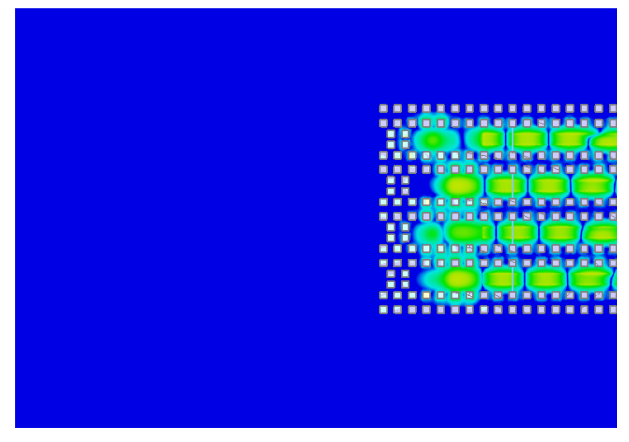

(b)

Fig. 21: Electric field at $30 \mathrm{GHz}$ of the mechanical phase shifter for a rotation angle of $\alpha=20^{\circ}$ on two levels: (a) the lower power divider and concentric phase-shifting GGW, and (b) the upper output RGW.

due to the constant increment of curvature radius between concentric phase-shifting GGW. Thereby, Fig. 20 only shows the phase shift for one pair of consecutive ports (between output ports 3 and 4) for each rotation angle $\alpha$.

The phase shift obtained agrees well with the expected phase difference according to (1). As we can see, just small oscillations around the expected values are observed in Fig. 20. The increment of the rotation angle produces a larger phase difference since the difference between path lengths is larger. The stability of the simulated phase shift versus frequency demonstrates the true-time-delay nature of the proposed phase 




(a)

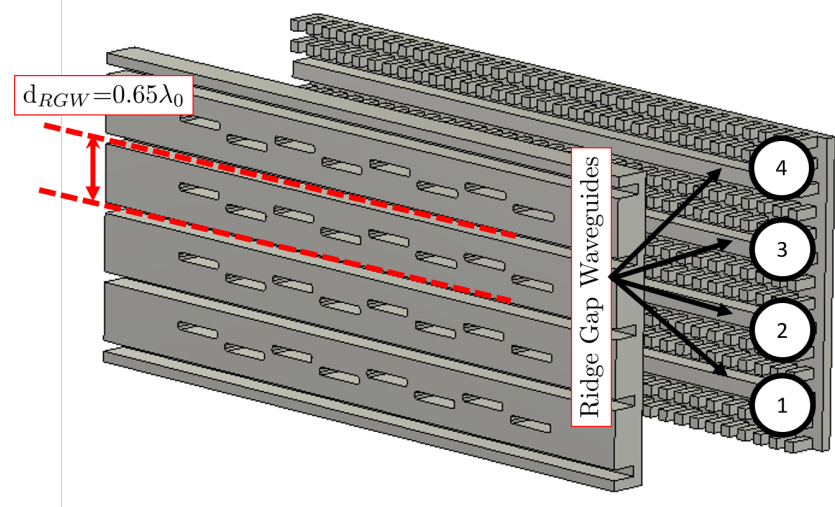

(b)

Fig. 22: 3D view of the slot array antenna: (a) complete structure, and (b) feeding and radiating layers separated.

TABLE III: Geometrical parameters of the slot array.

\begin{tabular}{|c|c||c|c|}
\hline Parameter & Value $(\mathrm{mm})$ & Parameter & Value $(\mathrm{mm})$ \\
\hline $\mathrm{w}_{R G W}$ & 1.5 & $\mathrm{~h}_{R G W}$ & 2.2 \\
\hline $\mathrm{d}_{R G W}$ & $6.5 \mathrm{~mm}$ & $\mathrm{~s}_{R G W}$ & 3.5 \\
\hline $\mathrm{w}_{c h}$ & 1 & $\mathrm{t}_{c h}$ & 2.5 \\
\hline $\mathrm{w}_{w g}$ & 5.5 & $\mathrm{t}_{\text {slot }}$ & 3.5 \\
\hline $\mathrm{d}_{\text {slots }}$ & $6.1 \mathrm{~mm}$ & $1_{\text {slots }}$ & 5.1 \\
\hline Air gap & 0.2 & offset & 0.45 \\
\hline
\end{tabular}

shifter. Note that the slight variations over the ideal phase behavior can be attributed to the small signal reflections at the different discontinuities.

To illustrate better the phase shift produced between output ports, Fig. 21 represents the instantaneous electric field at both levels of the mechanical phase shifter at $30 \mathrm{GHz}$. Fig. 21a shows the field in the lower rotatable block (1:4 power divider and concentric GGWs), and Fig. 21b shows the field in the upper fixed RGW. We can see the same phase in all output GGWs of the 1:4 power divider, but a small phase shift between the GGW at the end of the concentric phase-shifting GGWs, and between the output RGW.

\section{Slot ARRAY DESIGN}

The goal of the proposed mechanical phase shifter is to provide steering capabilities to an array antenna. This section



Fig. 23: Cross section of the slot array antenna.

describes a possible option for this antenna. In particular, an $8 \times 4$ slotted waveguide array antenna fed by four RGWs. These RGWs will be later connected to the mechanical phase shifter to steer the E-plane of the radiation pattern. The antenna is simply a proof-of-concept that can be extended later to larger arrays.

The slotted waveguide array, as shown in Fig. 22. exhibits 4 rows with 8 longitudinal slots. Each row is fed in series by a RGW, with the dimensions detailed in Section II-A. The conventional design method for resonant slot arrays [30] has been used, assuming that each slot behaves as a shunt admittance.

The design process starts by considering a resonant shunt slot array antenna formed by one single row. This result is later used as an initial guess to design the complete twodimensional array with 4 rows. As a result, the length and offset of each slot are adjusted to achieve a frequency response centered at the operation frequency band. The slots in each row present the same length and offset, thereby having a nearly uniform weight distribution. Two sets of slot dimensions are considered, one for the inner rows, and the other for the outer rows. This design process took into account the rounded corners of the slots, inherent to the milling manufacturing process used to fabricate the validation prototype.

The longitudinal separation between slots is $6.1 \mathrm{~mm}$, i.e., $0.61 \lambda_{0}$ at $30 \mathrm{GHz}$. The offset of the slots is around $0.45 \mathrm{~mm}$, with an alternate position with respect to longitudinal centerline in consecutive slots to grant an in-phase excitation. Recall that the separation between rows, i.e., the spacing of the slots in the XZ-plane, is $6.5 \mathrm{~mm}\left(0.65 \lambda_{0}\right)$.

Fig. 22 shows a 3D view of the complete slot array antenna. In particular, Fig. 22a shows the assembled structure, and Fig. 22b shows an exploded view of the two metal parts forming the antenna: the feeding and the radiating layers. As we can see in these figures, a longitudinal groove is added between rows. As commented in Section II-A, the internal coupling between rows is better than $50 \mathrm{~dB}$. The external mutual coupling, however, is still important since it corresponds to the non-directive cut of the radiation pattern. Such high mutual coupling between rows reduces the array bandwidth and scanning range. With the aim of decreasing slot coupling, a quarter wavelength deep groove acting as a choke is inserted between consecutive rows.

The most relevant dimensions of the radiating layer are illustrated in Fig. 23, including the thickness of the radiating 


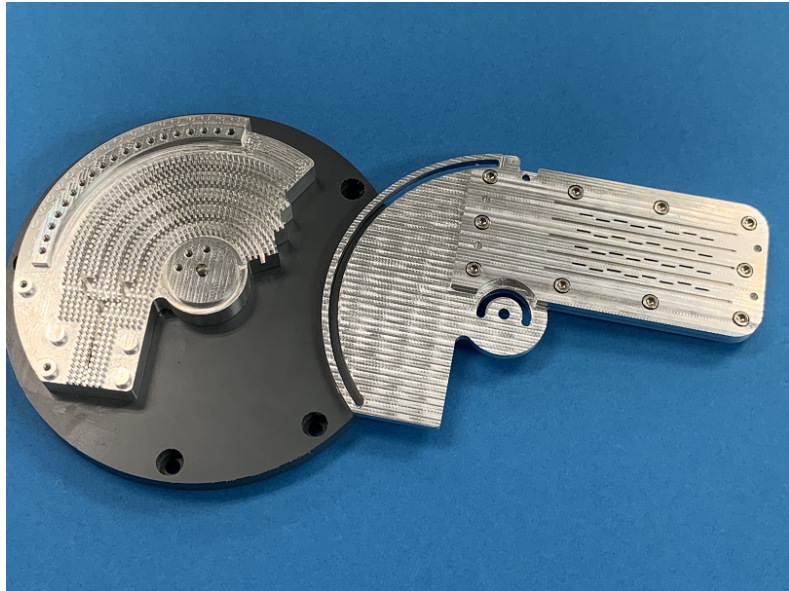

(a)

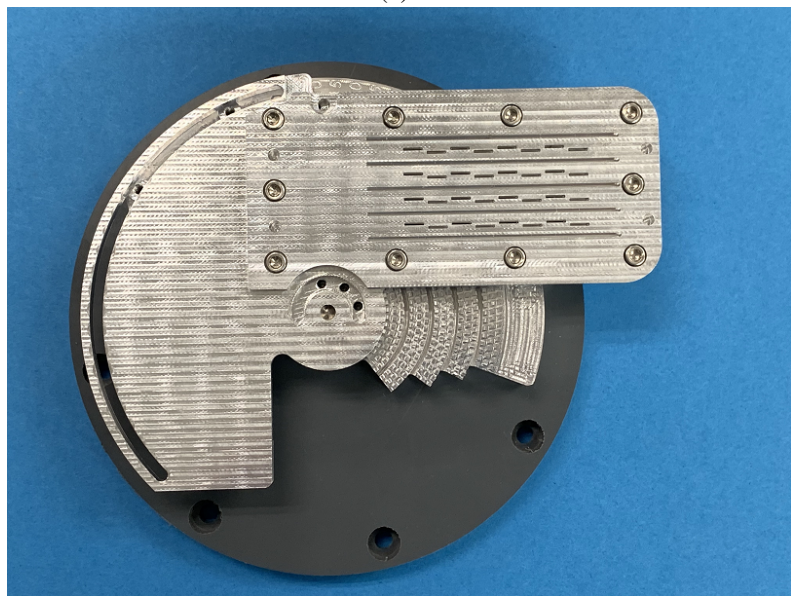

(c)

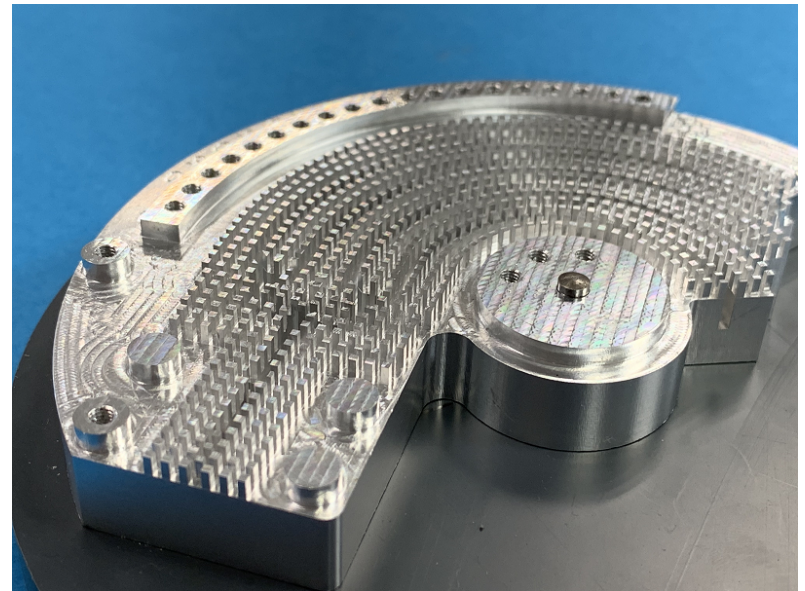

(b)

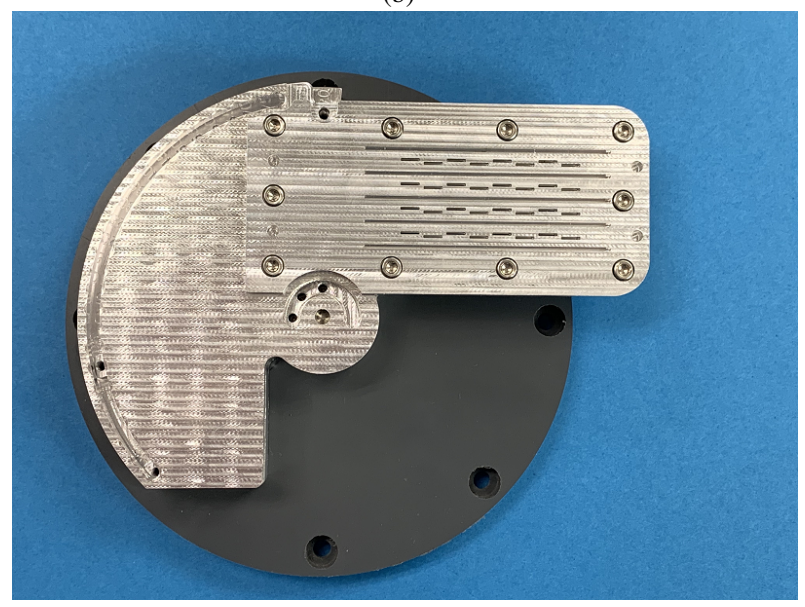

(d)

Fig. 24: Fabricated prototype of the complete mechanical phase shifter and the slot array antenna in GW technology: (a) lower-rotatable block (on the left) and upper-fixed block (on the right) with the slot array antenna, (b) closer view of the lower-rotatable block, (c) assembly for a rotation angle of $\alpha=0$, and (d) assembly for a rotation angle of $\alpha=90$

TABLE IV: Comparison between theoretical, simulated and measured pointing angle for different rotation angles from $0^{\circ}$ to $40^{\circ}$ in $10^{\circ}$ steps at different frequencies.

\begin{tabular}{c|ccc|ccc|ccc|}
\hline & \multicolumn{3}{|c}{ Theoretical } & \multicolumn{3}{c}{ Simulated } & \multicolumn{3}{c}{ Measured } \\
& $29.5 \mathrm{GHz}$ & $30.0 \mathrm{GHz}$ & $30.5 \mathrm{GHz}$ & $29.5 \mathrm{GHz}$ & $30.0 \mathrm{GHz}$ & $30.5 \mathrm{GHz}$ & $29.5 \mathrm{GHz}$ & $30.0 \mathrm{GHz}$ & $30.5 \mathrm{GHz}$ \\
\hline$\left(\alpha=0^{\circ}\right)$ & $0^{\circ}$ & $0^{\circ}$ & $0^{\circ}$ & $0^{\circ}$ & $0^{\circ}$ & $0.1^{\circ}$ & $0^{\circ}$ & $0^{\circ}$ & $0^{\circ}$ \\
$\left(\alpha=10^{\circ}\right)$ & $6.6^{\circ}$ & $6.8^{\circ}$ & $7.1^{\circ}$ & $6.5^{\circ}$ & $7^{\circ}$ & $6.6^{\circ}$ & $6.6^{\circ}$ & $6.6^{\circ}$ & $7.4^{\circ}$ \\
$\left(\alpha=20^{\circ}\right)$ & $13.3^{\circ}$ & $13.8^{\circ}$ & $14.3^{\circ}$ & $13.5^{\circ}$ & $13.5^{\circ}$ & $11.6^{\circ}$ & $12.1^{\circ}$ & $14.5^{\circ}$ & $9.9^{\circ}$ \\
$\left(\alpha=30^{\circ}\right)$ & $20.2^{\circ}$ & $20.9^{\circ}$ & $21.7^{\circ}$ & $21.5^{\circ}$ & $19.6^{\circ}$ & $22.4^{\circ}$ & $19.4^{\circ}$ & $19.8^{\circ}$ & $22.7^{\circ}$ \\
$\left(\alpha=40^{\circ}\right)$ & $27.4^{\circ}$ & $28.5^{\circ}$ & $29.6^{\circ}$ & $28^{\circ}$ & $27.1^{\circ}$ & $28.3^{\circ}$ & $26.6^{\circ}$ & $24.8^{\circ}$ & $30.4^{\circ}$ \\
\hline
\end{tabular}

layer $\left(t_{\text {slot }}=3.5 \mathrm{~mm}\right)$, and the dimensions of the chokes $\left(w_{c h}=1 \mathrm{~mm}\right.$ and $\left.h_{c h}=2.5 \mathrm{~mm}\right)$.

\section{EXPERIMENTAL RESULTS}

The slot array antenna and the mechanical phase shifter designed previously have been assembled to have a complete slot-array antenna with steering capabilities. The use of the same RGW technology, with the same dimensions, at the output ports of the mechanical phase shifter and the input ports of the slot array antenna, made possible the direct connection between the two devices. Fig. 1 shows an exploded view of the assembled slot array antenna and mechanical phase shifter.
The structure has been manufactured in-house in aluminum with a Datron M-25 Computer Numerical Control (CNC) Milling Machine. The weight of the complete structure is 330 g. Fig. 24 shows several pictures of the fabricated prototype. For validation purposes, concentric phase-shifting GGWs are longer than required for the fabricated slot array antenna.

Reference holes have been drilled along the contour of the phase-shifter to manually set the rotation angle. These holes, shown in Figs. 24a and b, are placed on 5-degree steps with respect to the rotation axis, leading to 19 discrete positions, from $0^{\circ}$ to $90^{\circ}$, for demonstration purposes.

Note that the chosen array spacing offers radiation patterns 

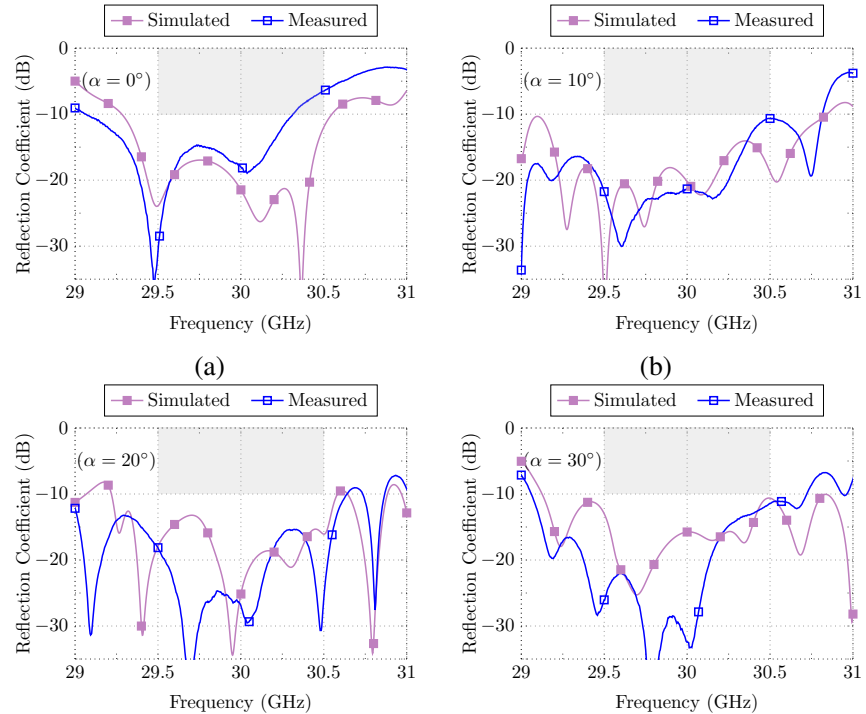

(c)

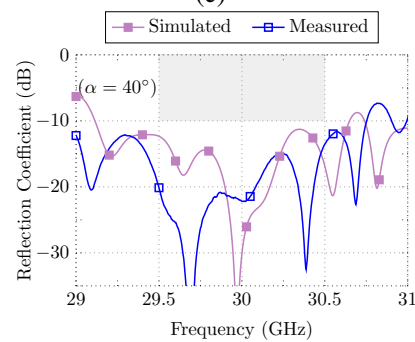

(e)

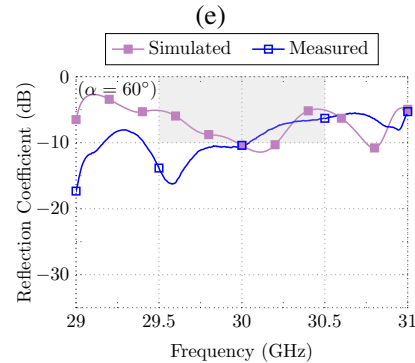

(g)

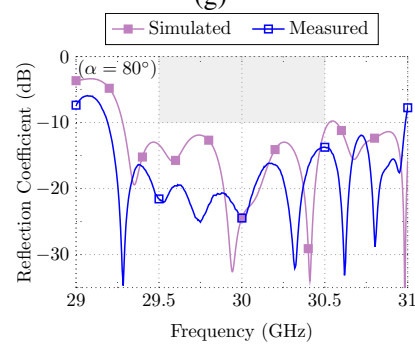

(i)

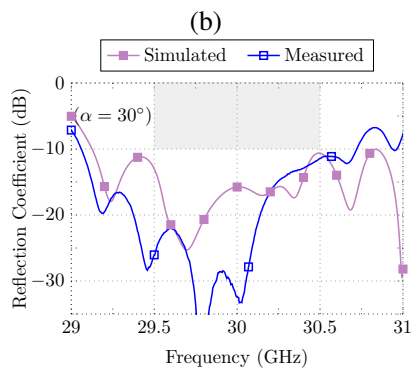

(d)


(h)



(j)

Fig. 25: Measured reflection coefficients for progressive rotating angles, in $10^{\circ}$ steps.

without grating lobes up to $\alpha=40^{\circ}$. Turning angles above $\alpha=40^{\circ}$ would dramatically increase sidelobes above acceptable values. This is due to the separation between the slotted waveguides in this particular case, $0.65 \lambda$.

In Fig. 24a, the two pieces are shown: the lower-rotatable block in GGW technology, and the upper-fixed block in RGW technology. This last block includes the slot array antenna
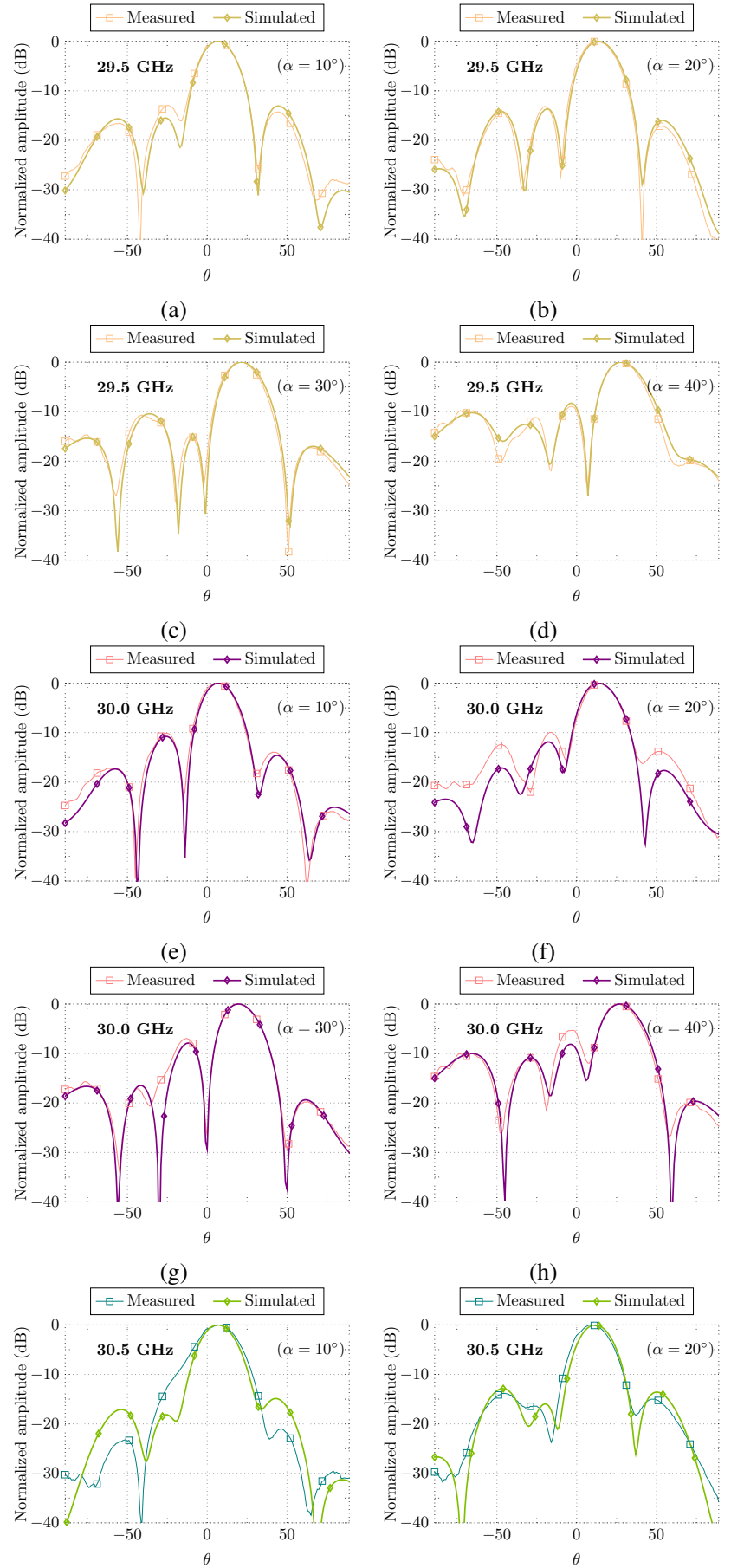

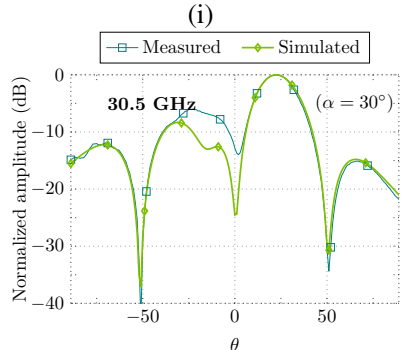

(k)



(1)
Fig. 26: Comparison between measured and simulated radiation patterns at 29.5 (a-d), 30 (e-h) and $30.5 \mathrm{GHz}(\mathrm{i}-\mathrm{l})$. 

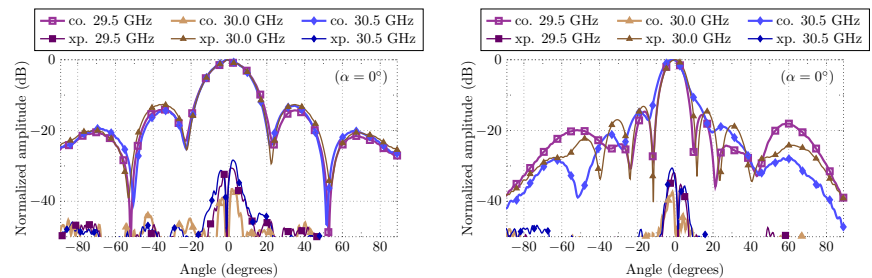

(a)

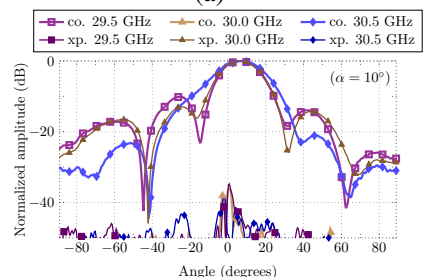

(c)



(e)

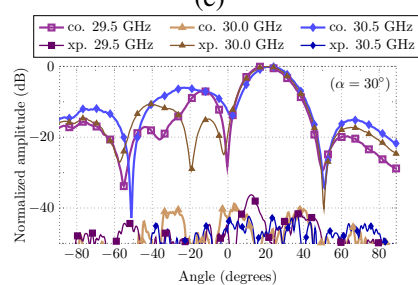

(g)

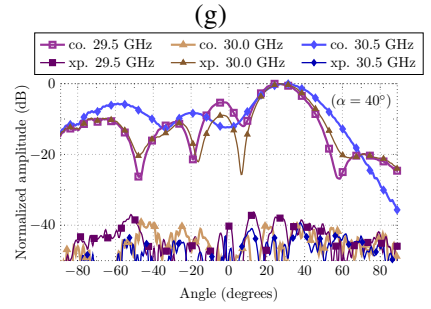

(i)

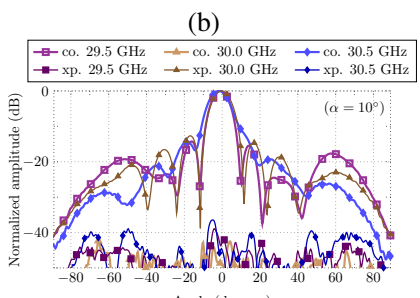

(d)

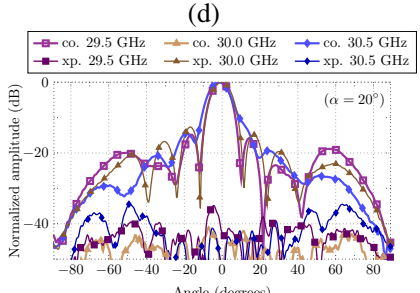

(f)

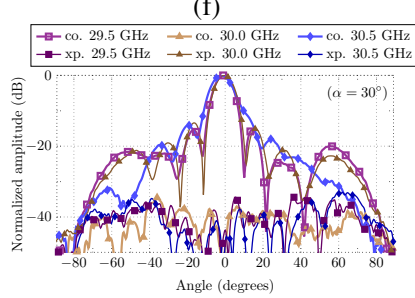

(h)

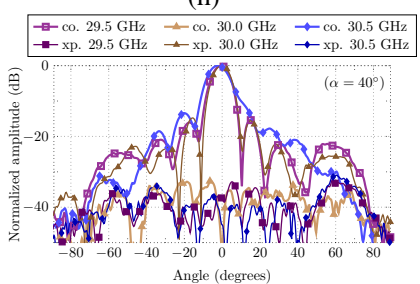

(j)

Fig. 27: Measured radiation patterns for different rotating angles of the mechanical phase shifter. Each pair of plots represents the E-plane (left) and $\mathrm{H}$-plane (right) for, respectively: $\alpha=\left[0^{\circ}, 10^{\circ}, 20^{\circ}, 30^{\circ}\right.$ and $\left.40^{\circ}\right]$.

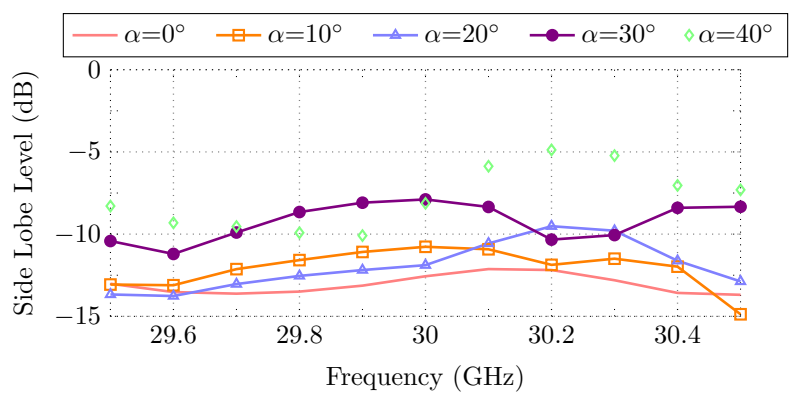

Fig. 28: Simulated E-plane sidelobe levels for different rotation angles.

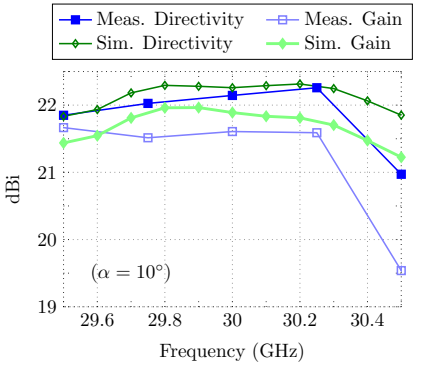

(a)

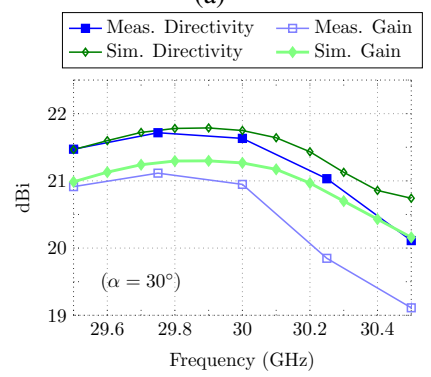

(c)



(b)

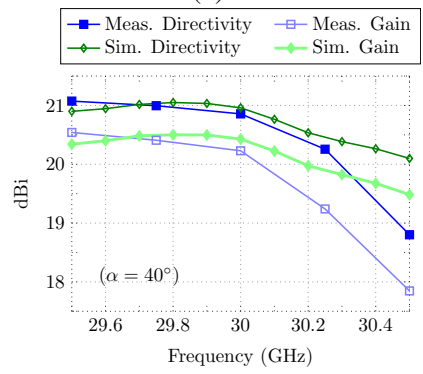

(d)
Fig. 29: Comparative between the measured directivity and gain for four rotation angles $\left(10^{\circ}, 20^{\circ}, 30^{\circ}\right.$ and $\left.40^{\circ}\right)$ and the corresponding simulations.

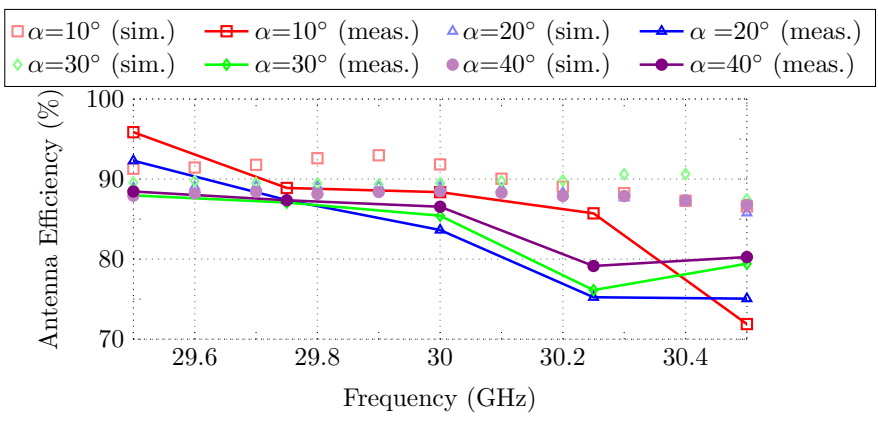

Fig. 30: Measured vs. simulated antenna efficiency for different rotation angles.

designed in previous section.

Fig. 24b illustrates with a closer view how the GGW are deployed in the lower block, from the input port to the concentric GGW. Note that the 1:4 power divider is fed from the back of the structure with a rectangular waveguide (WR28). A transition between the WR28 waveguide, and the GGW has been inserted to smoothly couple the energy between them. Figs. $24 \mathrm{c}$ and $24 \mathrm{~d}$ show an upper view of the complete structure in two different states, $\alpha=0^{\circ}$ and $\alpha=90^{\circ}$, respectively. As we can see, the upper block (with the slot array antenna) is fixed, no matter the rotation angle, while the lower block is rotated to produce the desired phase shift between the different rows of the array antenna.

The $\mathrm{S}_{11}$-parameter and the radiation pattern of the complete antenna have been measured to validate experimentally the manufactured prototype. Fig. 25 plots the measured reflection coefficient at the input port (WR28). The shaded area illustrates the operation frequency band, from $29.5 \mathrm{GHz}$ to $30.5 \mathrm{GHz}$. The gray threshold indicates a level of $-10 \mathrm{~dB}$. 
A worsening of the $\mathrm{S}_{11}$-parameter can be observed for $\alpha=60^{\circ}$ due to in-phase internal reflections in the concentric GGW.

The ten subfigures in Fig. 25 show the reflection coefficients for different rotation angles. The first plot (Fig. 25a) corresponds to an in-phase excitation of the 4 slotted waveguides, i.e. $\alpha=0^{\circ}$. The phase shifter is progressively rotated in steps of $\alpha=10^{\circ}$. The violet line represents the simulated $\mathrm{S}_{11^{-}}$ parameter, and the blue line the measured response. Despite not having a perfect agreement between simulations and measurements, the same trend is clearly observed in all the curves. The $-10 \mathrm{~dB}$ threshold is barely exceeded in only one case out of the 10 shown, which corresponds to a rotation angle of $60^{\circ}$.

Results in Table IV demonstrate how the experimental response approaches the simulated and theoretical patterns in terms of the steering obtained for every rotation angle.

In the light of these results, the maximum error between simulation and measurement has been observed at $30 \mathrm{GHz}$, where the deviation between the measured and the simulated results is $2.3^{\circ}$ for a rotation angle of $40^{\circ}$. The theoretical values are computed assuming ideal isotropic radiating elements an uniform amplitude, elements being spaced $6.5 \mathrm{~mm}$ apart. Considering all these aspects, the experimental values show a reasonable agreement with the expected results.

Fig. 26 provides a complete comparison between simulated and measured radiation patterns. Four rotation angles are shown $\left(10^{\circ}, 20^{\circ}, 30^{\circ}\right.$ and $\left.40^{\circ}\right)$ for the lower, middle and upper frequencies of the band. All radiation patterns show good agreement between them, although at $30.5 \mathrm{GHz}$ there are some minor discrepancies in the sidelobes. Also, the pointing angle is very similar between simulation and experimental results.

Fig. 27 shows the measured radiation patterns. In particular, the two main cuts of the patterns, E- and H-plane, are presented for rotation angles $\alpha$ from $0^{\circ}$ to $40^{\circ}$ in steps of $10^{\circ}$. When steering along the E-plane, the secondary lobe level rise. In general, the best performance is obtained at the central frequency, $30 \mathrm{GHz}$. In all cases, a high crosspolarization discrimination is obtained. It is also observed that an steering of $25^{\circ}$ is achieved with an approximate rotation angle of $\alpha=40^{\circ}$, as previously demonstrated in Table IV.

In fact, the final rotation angle is assumed to be $40^{\circ}$ since the prototype provides consistent measurements up to that rotation angle. If the rotation angle were increased above $40^{\circ}$, the steering angle would be larger than $25^{\circ}$ and grating lobes would be above acceptable levels. To illustrate this behavior, Fig. 28 shows how the levels of the sidelobe levels gradually rise as the angle of rotation increases.

Fig. 29 shows the directivity, gain of the measured antenna for several rotation angles. Also, a comparative graph between the measurement and simulated antenna efficiency for the same rotation angles of Fig. 29 is provided in Fig.30. High efficiency linked to full-metal antennas is verified. The lower the frequency, the higher the antenna efficiency, which reaches $90 \%$ levels. In the higher part of the working band, the efficiency remains above $70 \%$. Finally, for the sake of completeness, Table $\mathrm{V}$ provides the experimental results for directivity, gain, and antenna efficiency for the rotation angles interspersed from those previously plotted. There are fluctuations depending
TABLE V: Measured antenna performance

\begin{tabular}{lccccc}
\hline & Freq. & $\alpha=5^{\circ}$ & $\alpha=15^{\circ}$ & $\alpha=25^{\circ}$ & $\alpha=35^{\circ}$ \\
\hline \multirow{3}{*}{ Directivity (dBi) } & $29.5 \mathrm{GHz}$ & 22.13 & 21.81 & 21.49 & 21.29 \\
& $30.0 \mathrm{GHz}$ & 22.29 & 22.17 & 21.85 & 21.20 \\
& $30.5 \mathrm{GHz}$ & 21.91 & 20.52 & 21.74 & 20.05 \\
\hline \hline \multirow{3}{*}{ Gain (dBi) } & $29.5 \mathrm{GHz}$ & 21.99 & 21.35 & 21.11 & 20.82 \\
& $30.0 \mathrm{GHz}$ & 21.88 & 21.63 & 21.22 & 20.12 \\
& $30.5 \mathrm{GHz}$ & 20.93 & 19.35 & 20.95 & 18.63 \\
\hline \hline \multirow{3}{*}{ Antenna Eff. (\%) } & $29.5 \mathrm{GHz}$ & 92.13 & 88.86 & 91.74 & 89.65 \\
& $30.0 \mathrm{GHz}$ & 91.01 & 88.09 & 86.33 & 78.08 \\
& $30.5 \mathrm{GHz}$ & 79.88 & 76.40 & 83.25 & 72.05 \\
\hline
\end{tabular}

on rotation angle and working frequency, but these data corroborate the trend shown graphically in Fig. 29, with high efficiency levels, directivity around $22 \mathrm{dBi}$, and gain close to $21 \mathrm{dBi}$.

\section{Conclusions}

This work presents a fully-metallic mechanically-steerable slot array antenna in gap-waveguide technology. The whole antenna consist in two pieces: a lower-rotatable block, which changes the length of the lower concentric GGW, and an upper-fixed block, where the slot array antenna, fed by RGWs, is located. The main advantage of the steering mechanism presented is that it only requires the mechanical rotation of an internal part of the structure, leaving fixed the slot array itself. Such rotation movement can be implemented by relativelty simple engines, especially compared to those of conventional positioning systems.

The experimental measurements reported validate the proposed concept, having obtained steering angles of up to $25^{\circ}$ with gain levels of $21 \mathrm{dBi}$. It is worth noting the high efficiency and the good matching achieved for all its possible rotation angles in a bandwidth from $29.5 \mathrm{GHz}$ to $30.5 \mathrm{GHz}$. Peak efficiencies above $90 \%$ have been reported thanks to the fact that the antenna has been constructed entirely in aluminum. Note that the limitation in bandwidth is mostly attributable to the shunt slot array performance.

The proposed steering mechanism adopting variable-length arc-shaped gap waveguides has demonstrated to be a feasible option to attain beam-steerable slot arrays. Its architecture is completely scalable to much larger arrays and its inherent true-time-delay feature grants a wideband performance of the phase-shifting scheme. Future research should explore how to exploit all the benefits described here. Yet, most of the potential enhancement will come from the modification of the radiating slot array. Closer row spacing as well as the reduction of mutual coupling between elements will naturally bring wider scanning ranges.

The adoption of alternatives to resonant slot arrays will also entail larger operation bandwidths. The proposed solution can also be easily adapted to design low-sidelobe patterns along both main planes. Finally, several modifications of the lower rotatable piece can be foreseen to reduce the size of the entire structure, such as adopting a double-layer folded architecture for the lower piece so that it all remains hidden below the radiating layer. 


\section{ACKNOWLEDGMENT}

This work has been supported by the Spanish Ministry of Science, Innovation and Universities (Ministerio de Ciencia, Innovacion y Universidades) under project TEC2016-79700C2-1-R.

\section{REFERENCES}

[1] J. I. Herranz-Herruzo, A. Valero-Nogueira, M. Ferrando-Rocher, B. Bernardo, A. Vila, and R. Lenormand, "Low-cost Ka-band switchable RHCP/LHCP antenna array for mobile SATCOM terminal," IEEE Trans. Antennas Propag., vol. 66, no. 5, pp. 2661-2666, 2018.

[2] F. Tabarani, L. Boccia, T. Purtova, A. Shamsafar, H. Schumacher, and G. Amendola, "0.25- $\mu \mathrm{m}$ BiCMOS system-on-chip for $\mathrm{K}-/ \mathrm{Ka}$-Band satellite communication transmit-receive active phased arrays," IEEE Trans. Microw. Theory Techn., vol. 66, no. 5, pp. 2325-2339, 2018.

[3] L. Di Palma, A. Clemente, L. Dussopt, R. Sauleau, P. Potier, and P. Pouliguen, "Circularly-polarized reconfigurable transmitarray in kaband with beam scanning and polarization switching capabilities," IEEE Trans. Antennas Propag., vol. 65, no. 2, pp. 529-540, 2017.

[4] E. B. Lima, S. A. Matos, J. R. Costa, C. A. Fernandes, and N. J. G. Fonseca, "Circular polarization wide-angle beam steering at Ka-Band by in-plane translation of a plate lens antenna," IEEE Trans. Antennas Propag., vol. 63, no. 12, pp. 5443-5455, 2015.

[5] S. A. Matos, E. B. Lima, J. S. Silva, J. R. Costa, C. A. Fernandes, N. J. G. Fonseca, and J. R. Mosig, "High gain dual-band beam-steering transmit array for satcom terminals at Ka-Band," IEEE Trans. Antennas Propag., vol. 65, no. 7, pp. 3528-3539, 2017.

[6] H. Wang, Z. Wang, Z. Wu, and Y. Zhang, "Beam-scanning lens antenna based on elliptical paraboloid phase distribution metasurfaces," IEEE Antennas Wireless Propag. Lett., vol. 18, no. 8, pp. 1562-1566, 2019.

[7] P. Qin, L. Song, and Y. J. Guo, "Beam steering conformal transmitarray employing ultra-thin triple-layer slot elements," IEEE Trans. Antennas Propag., vol. 67, no. 8, pp. 5390-5398, 2019.

[8] N. Gagnon and A. Petosa, "Using rotatable planar phase shifting surfaces to steer a high-gain beam," IEEE Trans. Antennas Propag., vol. 61, no. 6, pp. 3086-3092, 2013.

[9] M. U. Afzal and K. P. Esselle, "Steering the beam of medium-tohigh gain antennas using near-field phase transformation," IEEE Trans. Antennas Propag., vol. 65, no. 4, pp. 1680-1690, 2017.

[10] A. A. Baba, R. M. Hashmi, K. P. Esselle, M. Attygalle, and D. Borg, "A millimeter-wave antenna system for wideband 2-D beam steering," IEEE Trans. Antennas Propag., vol. 68, no. 5, pp. 3453-3464, 2020.

[11] N. K. Host, C. Chen, J. L. Volakis, and F. A. Miranda, "Ku-band traveling wave slot array scanned via positioning a dielectric plunger," IEEE Trans. Antennas Propag., vol. 63, no. 12, pp. 5475-5483, 2015.

[12] A. Ghasemi and J. Laurin, "A continuous beam steering slotted waveguide antenna using rotating dielectric slabs," IEEE Trans. Antennas Propag., vol. 67, no. 10, pp. 6362-6370, 2019.

[13] E. Gandini, M. Ettorre, M. Casaletti, K. Tekkouk, L. Le Coq, and R. Sauleau, "SIW slotted waveguide array with pillbox transition for mechanical beam scanning," IEEE Antennas Wireless Propag. Lett., vol. 11, pp. 1572-1575, 2012.

[14] H. Qiu, X. Yang, Y. Yu, T. Lou, Z. Yin, and S. Gao, "Compact beam-scanning flat array based on substrate-integrated waveguide," IEEE Trans. Antennas Propag., vol. 68, no. 2, pp. 882-890, 2020.

[15] X. Lu, S. Gu, X. Wang, H. Liu, and W. Lu, "Beam-scanning continuous transverse stub antenna fed by a ridged waveguide slot array," IEEE Antennas Wireless Propag. Lett., vol. 16, pp. 1675-1678, 2017.

[16] K. Tekkouk, J. Hirokawa, R. Sauleau, and M. Ando, "Wideband and large coverage continuous beam steering antenna in the $60-\mathrm{GHz}$ band," IEEE Trans. Antennas Propag., vol. 65, no. 9, pp. 4418-4426, 2017.

[17] P.-S. Kildal, E. Alfonso, A. Valero-Nogueira, and E. Rajo-Iglesias, "Local metamaterial-based waveguides in gaps between parallel metal plates," IEEE Antennas Wireless Propag. Lett., vol. 8, pp. 84-87, 2009.

[18] H. Kirino and K. Ogawa, "A $76 \mathrm{GHz}$ multi-layered phased array antenna using a non-metal contact metamaterial waveguide," IEEE Trans. Antennas Propag., vol. 60, no. 2, pp. 840-853, 2012.

[19] X. Fang, Y. Huang, and G. Wen, "A low-cost mechanical beam scanning waveguide slot antenna array," in 2017 IEEE International Symposium on Antennas and Propagation USNC/URSI National Radio Science Meeting, 2017, pp. 2603-2604.
[20] M. Ferrando-Rocher, J. I. Herranz-Herruzo, A. Valero-Nogueira, B. Bernardo-Clemente, A. U. Zaman, and J. Yang, " $8 \times 8 k a$-band dual-polarized array antenna based on gap waveguide technology," IEEE Trans. Antennas Propag., vol. 67, no. 7, pp. 4579-4588, 2019.

[21] A. Vosoogh, P. Kildal, and V. Vassilev, "Wideband and high-gain corporate-fed gap waveguide slot array antenna with etsi class ii radiation pattern in $v$-band," IEEE Trans. Antennas Propag., vol. 65, no. 4, pp. 1823-1831, 2017.

[22] D. Sánchez-Escuderos, J. I. Herranz-Herruzo, M. Ferrando-Rocher, and A. Valero-Nogueira, "Mechanical phase shifter in gap-waveguide technology," in 2020 14th European Conference on Antennas and Propagation (EuCAP). IEEE, 2020, pp. 1-5.

[23] D. Sánchez-Escuderos, J. I. Herranz-Herruzo, and A. Valero-Nogueira, "Desfasador mecánico basado en guías gap sin contacto," 2019, PCT/ES2020/070296.

[24] M. Ferrando-Rocher, A. Valero-Nogueira, J. I. Herranz-Herruzo, A. Berenguer, and B. Bernardo-Clemente, "Groove gap waveguides: A contactless solution for multilayer slotted-waveguide array antenna assembly," in 2016 10th European Conference on Antennas and Propagation (EuCAP). IEEE, 2016, pp. 1-4.

[25] E. Rajo-Iglesias, M. Ferrando-Rocher, and A. U. Zaman, "Gap waveguide technology for millimeter-wave antenna systems," IEEE Commun. Mag., vol. 56, no. 7, pp. 14-20, 2018.

[26] M. Ferrando Rocher, "Gap waveguide array antennas and corporate-feed networks for mm-wave band applications," Ph.D. dissertation, 2019.

[27] E. Rajo-Iglesias and P.-S. Kildal, "Groove gap waveguide: A rectangular waveguide between contactless metal plates enabled by parallel-plate cut-off," in 4th European Conference on Antennas and Propagation. IEEE, 2010, pp. 1-4.

[28] C. M. Studio, "3d em simulation software," Computer Simulation Technology, 2017.

[29] A. J. Sáez, A. Valero-Nogueira, J. I. Herranz, and B. Bernardo, "Singlelayer cavity-backed slot array fed by groove gap waveguide," IEEE Antennas Wireless Propag. Lett., vol. 15, pp. 1402-1405, 2015.

[30] R. C. Johnson and H. Jasik, "Antenna engineering handbook," $m g h$, 1984.

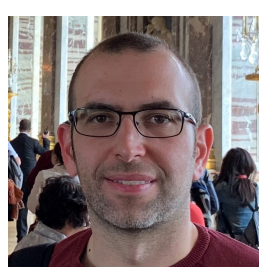

Daniel Sánchez Escuderos (S'05-M'09) was born in Vila Real, Spain, in 1980. He received the M.S. degree in telecommunications engineering and the Ph.D. degree in telecommunications engineering from the Universitat Politècnica de València (UPV), Valencia, Spain, in 2004 and 2009, respectively. Since 2005 , he has been with the Institute of Telecommunications and Multimedia Applications, UPV. In 2009, he became a Post-Doctoral Researcher under the framework of several research activities. From 2009 to 2014, he was involved in the National Research Project in terahertz technology. Since 2014, he has been collaborating in several European projects supported by the European Space Agency and national research projects. His current research interests include antenna measurements, frequency-selective surface (FSS) structures, millimeter- and submillimeter-wave technology, gap-waveguide technology, and microwave filters. Dr. Sánchez-Escuderos has been a member of the IEEE Antennas and Propagation Society and the session chair at several scientific conferences since 2005. He was a recipient of an FPI National Scholarship in support of his Ph.D. studies in 2005.

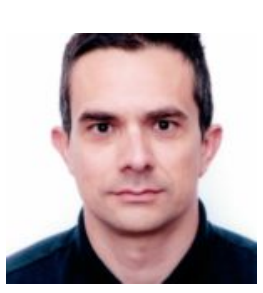

Jose Ignacio Herranz-Herruzo. (M'04) was born in Valencia, Spain, in 1978. He received the Degree in telecommunication engineering from the Universitat Politècnica de València, Valencia, in 2002, where he is currently pursuing the Ph.D. degree. In 2002, he joined the Departamento de Comunicaciones, Universitat Politècnica de València, where he is currently an Associate Professor. His current research interests include the optimization of waveguide slot array antennas and efficient computational methods for planar structure. 




Miguel Ferrando-Rocher (S'15-M'19) was born in Alcoy, Spain. He received the M.Sc. and Ph.D. degrees in telecommunication engineering from the Universitat Politècnica de València (UPV), Valencia, Spain, in 2012 and 2018, respectively. In 2012, he joined the Complex Radiation Systems Team, Institute of Electronics and Telecommunications Rennes, France, as a Researcher, where he was involved in reflectarray antennas for satellite applications in collaboration with Thales Alenia Space, Paris, France. In 2016, he joined the Chalmers University of Technology, Gothenburg, Sweden, as a Guest Researcher. Since 2013, he has been with the Antennas and Propagation Laboratory (APL) of the Institute of Telecommunications and Multimedia Applications (iTEAM) of UPV. He is currently Assistant Professor at the University of Alicante, in the Department of Physics, Systems Engineering and Signal Theory. His research activity is also developed in the Microwave and Electromagnetism Group (GMECA). His research interests include satellite communication (SATCOM) on-the-move, high-gain antennas and arrays, gap waveguide (GW) technology, and millimeter-wave components. Dr. Ferrando-Rocher was a recipient of the AIRBUS Defence and Space Award in 2019, the Extraordinary Doctoral Thesis Award from the Polytechnic University of Valencia (Spain) in 2020 and the URSI Conference Best Student Paper Award in 2017. He received an Erasmus Grant to study at Ghent University, Ghent, Belgium, in 2010.

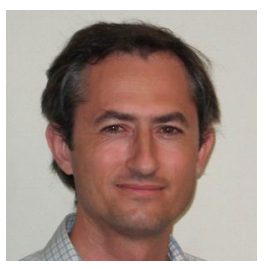

Alejandro Valero-Nogueira (S'92-M'97-SM'09) was born in Madrid, Spain, in 1965. $\mathrm{He}$ received the Degree in telecommunication engineering from the Universidad Politécnica de Madrid, Madrid, in 1991 and the Ph.D. degree in telecommunication from the Universitat Politècnica de València, Valencia, Spain, in 1997. In 1992, he joined the Departamento de Comunicaciones, Universitat Politècnica de València, where he is currently an Full Professor. During 1999, he was on leave at the ElectroScience Laboratory, The Ohio State University, Columbus, $\mathrm{OH}$, USA, where he was involved in fast solution methods in electromagnetics (EMs) and conformal antenna arrays. His current research interests include computational EMs, waveguide slot arrays, gap waveguides $(\mathrm{GW})$, theory of characteristic modes, and automated antenna design procedures. 